\title{
A semi-explicit algorithm for the reconstruction of $3 D$ planar cracks
}

Tarek Bannour , Amel Ben AbDA , Mohamed JaOuA

$$
\mathrm{N}^{\circ} \mathbf{3 0 6 3}
$$

December 1996

THÈME 4 



\title{
A semi-explicit algorithm for the reconstruction of $3 \mathrm{D}$ planar cracks
}

\author{
Tarek Bannour * Amel Ben ABda ${ }^{\dagger}$, Mohamed JaOuA ${ }^{\ddagger}$ \\ Thème 4 - Simulation et optimisation \\ de systèmes complexes \\ Projet Mostra
}

Rapport de recherche $\mathrm{n}^{\circ} 3063$ - December 1996 - 21 pages

\begin{abstract}
This paper deals with a semi-explicit algorithm to reconstruct $2 \mathrm{D}$ segment cracks, or $3 \mathrm{D}$ planar cracks, in the framework of overspecified boundary data. The algorithm is based on the reciprocity gap concept, introduced by S. Andrieux and A. Ben Abda, which provides explicitely the line (or the plane) support of the cracks. A numerical reconstruction of the cracks, which are actually the support of the solution jump across this plane, is then performed by computing the Fourier expansion of the solution jump itself. After the numerical analysis of the method, some numerical results are presented and commented.
\end{abstract}

Key-words: geometrical inverse problems, crack detection, reciprocity gap, error analysis, semiexplicit algorithm.

(Résumé : tsvp)

E-mail : mohamed.jaoua@inria.fr

This work was partially achieved during a visit of the second author at INRIA, supported by the french-tunisian cooperation within the CMCU research program entitled Méthodes de simulation numérique. The third author's research is partially supported, during his sabbatic leave at INRIA, by a french governement's MENESR-DRIC grant.

* ENIT-LAMAP, BP 37, 1002 Tunis-Belvédère, Tunisia

${ }^{\dagger}$ ENIT-LAMAP \& IPEST, BP 51, 2070 La Marsa, Tunisia

$\ddagger$ ENIT-LAMAP \& INRIA, projet Mostra, Domaine de Voluceau, Rocquencourt, 78153 Le Chesnay cedex, France 


\section{Un algorithme semi-explicite de reconstruction de fissures planes 3D}

Résumé : On présente dans ce travail un algorithme semi-explicite de reconstruction de fissures droites (en 2D) ou planes (en 3D) grâce à des mesures effectuées sur la frontière. L'algorithme est basé sur le concept d'écart à la réciprocité, introduit [par S. Andrieux et A. Ben Abda, qui permet de déterminer explicitement la ligne (resp. le plan) portant la ou les fissures. La reconstruction numérique de ces dernières, qui constituent en fait le support du saut de la solution à travers la ligne (resp. le plan), est ensuite obtenue en calculant le développement en série de Fourier du saut. L'analyse numérique de la méthode est ensuite effectuée, suivie de la présentation de quelques résultats numériques commentés.

Mots-clé : problèmes inverse géométriques, détection de fissures, écartr à la réciprocité, analyse d'erreur, algorithme semi-explicite. 


\section{Introduction}

In this work, we are interested in the reconstruction of line segment cracks (in $2 \mathrm{D}$ situations), or planar cracks (in 3D situations) by overspecified boundary data. These problems are related to non destructive electric or thermic control of materials.

In this area, there are quite a few theoretical works, and almost all of them deal with $2 \mathrm{D}$ cases : a uniqueness result for a buried crack has been investigated by Friedman and Vogelius [16, 1989], and stability results of logarithmic type have been obtained by Alessandrini $[2,1993]$. For the case of emerging cracks at an a priori known point of the boundary, a uniqueness result and a local Lipschitzstability one have been proved in $[9,1996]$. In the case of a family of emerging cracks, a uniqueness result has been proved in $[14,1995]$. As for $3 \mathrm{D}$ situations, only very few uniqueness results exist, and they all assume the knowledge of all the possible measurements, namely the full Neumann-to-Dirichlet operator (see Eller $[15,1996]$ and references therein).

An efficient numerical reconstruction algorithm - for interior segment cracks - has been presented by Santosa and Vogelius [19, 1990], and a more recent work by Elcrat and Isakov [14, 1995] gives an inversion algorithm to recover a family of surface cracks.

In the particular case of 3D-planar (or 2D-line segment) cracks, S. Andrieux and A. Ben Abda introduced in $[6,1993][7,1996]$ the reciprocity gap concept, and proved that such cracks can be completely determined, provided that complete data are available on the external boundary of the body. Moreover, they gave inversion formulae which determine explicitely the plane containing the cracks, and proved that the full reconstruction is possible. The proof of the latest result turns to be constructive, and the semi-explicit algorithm is actually built upon it.

In section 2, the results used to construct the numerical algorithm and to run the trials are recalled. The semi-explicit algorithm is described and studied in section 3, while the numerical results and comments are presented in section 4.

\section{The inversion process.}

For the reader's convenience, we recall in this section the results related to the reciprocity gap concept [7] that we shall use to built the numerical semi-explicit algorithm and to run the trials.

In this section, we first derive the reciprocity gap notion and the functional associated to it. Then, we use this functional to establish the formulae for the identification of the plane containing the cracks, a complete identification result, and another formula for the determination of an emerging crack length in $2 \mathrm{D}$ situations.

\subsection{Uniqueness results for 3D-planar cracks.}

Let $\Omega$ denote the 3D bounded domain occupied by the body,and $\partial \Omega$ its external boundary, which we shall assume to be $\mathcal{C}^{2}$. The body is supposed to contain one or more co-planar cracks $\sigma \in \Pi$, where ( $\Pi$ ) is the affine plane in $R^{3}$ containing all the cracks. We denote by $\Omega_{\sigma}=\Omega \backslash \sigma$ the defective (cracked) domain. The affine space is equipped with a direct orthonormal frame $\left(O, e_{1}, e_{2}, e_{3}\right)$. Denoting by $\left(x_{1}, x_{2}, x_{3}\right)$ the corresponding cartesian coordinates system, the plane equation will be given by :

$$
n_{1} x_{1}+n_{2} x_{2}+n_{3} x_{3}+c=0
$$

where $\vec{N}=\left(n_{1}, n_{2}, n_{3}\right)$ is a unit normal vector to $(\Pi)$.

Let us now denote by $\phi$ a given heat flux on $\partial \Omega$, satisfying $\phi \not \equiv 0$ and $\int_{\partial \Omega} \phi=0, \phi \in H^{-\frac{1}{2}}(\partial \Omega)$ (in practise, $\phi$ will be chosen to be piecewise continuous). Consider then the following steady state electrical (or heat conduction) problem for a homogeneous medium :

$\mathrm{RR} \mathrm{n}^{\circ} 3063$ 


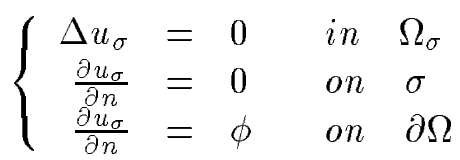

Since the solution of (2) is unique up to a constant, the condition $\int_{\partial \Omega} u_{\sigma}$ is added in order to insure the uniqueness. We assume in the following that this solution has been measured on the whole external boundary $\partial \Omega$, and we shall denote by $f$ this measure.

\subsection{The reciprocity gap concept.}

In the general framework of flaw determination, one can intuitively feel that some insight into the problem may be gained by comparing the actual response of the body (a flux being prescribed) to the one given by a safe body with the same characteristics.

The first idea is to examine both state fields. This cannot be useful, so far as these fields are defined on two different domains (the safe one, and the defective one), and the field corresponding to the defective body is only known by its Cauchy conditions on the external boundary. One has then to carry all the available information on the known part of the body. To this end, the Maxwell-Betti reciprocity principle (in elastostatics) provides the idea, which extends in fact to any symmetric operator. For sake of simplicity, we shall present it in the case of elliptic operators. The variational formulation of such problems can be settled as follows :

$$
\left\{\begin{array}{l}
\text { Find } u \text { in } H, \text { such that }: \\
a(u, v)=L(v) \text { for any } v \in H
\end{array}\right.
$$

where $H$ is a Hilbert space, $a$ a bilinear, symmetric and coercive form, continuous on $H \times H$, and $L$ a linear continuous form on $H . L_{1}$ and $L_{2}$ being two different linear forms defined on $H$, let us consider the two corresponding problems $(i=1,2)$ :

$$
\left\{\begin{array}{l}
\text { Find } u_{i} \text { in } H, \text { such that : } \\
a\left(u_{i}, v\right)=L_{i}(v) \text { for any } v \in H
\end{array}\right.
$$

Then, choosing $v=u_{2}$ as a test function for the first problem, and $v=u_{1}$ for the second one, we derive that $L_{1}\left(v_{2}\right)=L_{2}\left(v_{1}\right)$. This is the reciprocity principle, due to the symmetry of $a$. Let us now reconsider the particular case of the Laplace equation, and let $\tilde{u}$ be the solution of the following problem in the safe body :

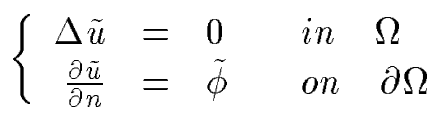

where $\tilde{\phi}$ is some flux verifying $\int_{\partial \Omega} \tilde{\phi}=0$. Denote by $\tilde{f}$ the measured temperature on the external boundary $\left(\left.\tilde{u}\right|_{\partial \Omega}=\tilde{f}\right)$, and consider the quantity :

$$
\int_{\partial \Omega}(\phi \tilde{f}-\tilde{\phi} f)
$$

According to the reciprocity principle recalled above, this quantity vanishes if the domain is safe. The reciprocity gap is then naturally defined, for any harmonic function $v \in H^{1}(\Omega)$, as follows : 


$$
R G_{[\phi, f]}(v)=\int_{\partial \Omega} \phi v-\frac{\partial v}{\partial n} f
$$

Equation (6) defines then a linear form on the space $H=\left\{v \in H^{1}(\Omega)\right.$ such that $\Delta v=0$ in $\left.\Omega\right\}$. By using Green's formula on the domain $\Omega_{\sigma}$, we get :

$$
R G_{[\phi, f]}(v)=\int_{\sigma}\left[u_{\sigma}\right] \frac{\partial v}{\partial N}
$$

where $\left[u_{\sigma}\right]$ denotes the jump of the $u_{\sigma}$ across $\sigma$, with respect to the direction of the normal $N$. The following result then holds.

Proposition 1 (Determination of a normal vector to the plane) Denote by $\chi_{k}$ the mapping $x \longmapsto x_{k}$, and let $L_{k}=R G_{[\phi, f]}\left(\chi_{k}\right)$. If $\phi$ has been chosen in such a way that $\int_{\sigma}\left[u_{\sigma}\right] \neq 0$, then the components of the unit normal to the plane (П) containing the cracks $\sigma$ are given by :

$$
n_{k}=\frac{L_{k}}{\sqrt{L_{1}^{2}+L_{2}^{2}+L_{3}^{2}}} \text { for } k=1,2,3
$$

Furthermore, one has :

$$
\left|\int_{\sigma}\left[u_{\sigma}\right]\right|=\sqrt{L_{1}^{2}+L_{2}^{2}+L_{3}^{2}}
$$

Proof : It is straightforward from expressions (6) and (7) of the reciprocity gap applied to the harmonic functions $\chi_{k}, k=1,2,3$.

A unit normal vector $\vec{N}$ having been determined by the previous proposition, consider a new direct orthonormal basis $(\vec{T}, \vec{V}, \vec{N})$, and denote by $\left(X_{1}, X_{2}, X_{3}\right)$ the corresponding cartesian coordinates system with origin $O$. The equation of plane (П) in this system will be :

$$
X_{3}-c=0
$$

Under the same assumption $\int_{\sigma}\left[u_{\sigma}\right] \neq 0$, the following result completes the localization of the plane (II) by determining the constant $c$.

Proposition 2 (Determination of the constant) The constant $c$ determining the plane (П) is given by :

$$
c=\frac{R G_{[\phi, f]}(p)}{\sqrt{L_{1}^{2}+L_{2}^{2}+L_{3}^{2}}}
$$

where :

$$
p\left(X_{1}, X_{2}, X_{3}\right)=\frac{X_{3}^{2}-X_{2}^{2}}{2}
$$

Proof :It is also based on the reciprocity gap applied to some specisl function, as shown in [7] 


\subsection{The complete identification of the cracks.}

At this stage, the plane (П) containing the cracks is entirely, and explicitely, determined.

A constructive method is now proposed to achieve the cracks identification. Once again, the reciprocity gap is the basic tool. Based on its two expressions (6) and (7), the identification of $\sigma$ is performed by interpreting $\left[u_{\sigma}\right]$ as a linear form on $L^{2}(S), S$ being some square domain of the plane (I) containing the cracks.

Consider now a new frame $\left(O^{\prime}, \vec{T}, \vec{V}, \vec{N}\right)$ obtained by a simple translation, such that the new origin $O^{\prime}$ belongs to $(\Pi)$. Let $d=\operatorname{diam}(\Omega)$, and $\mathcal{W}$ be some open "big box" containing $\Omega$. Setting $d=2$ does not reduce the generality, and then for example :

$$
\mathcal{W}=] a_{1}-1, a_{1}+1[\times] a_{2}-1, a_{2}+1[\times] a_{3}-1, a_{3}+1[
$$

where $\left(a_{1}, a_{2}, a_{3}\right)$ are the coordinates of some appropriate interior point to $\Omega$, with respect to the frame $\left(O^{\prime}, \vec{T}, \vec{V}, \vec{N}\right)$.

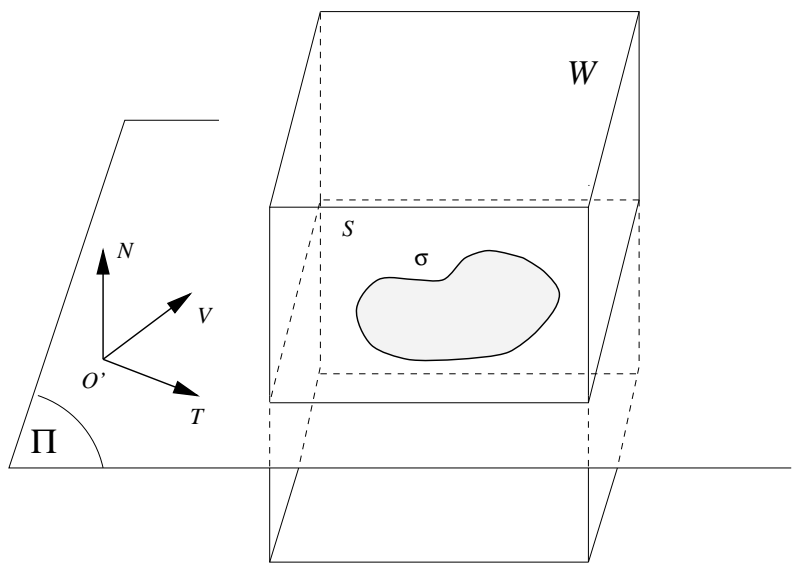

Figure 1: The crack laying in the determined plane

Let us choose $S=\Pi \cap \mathcal{W}$, and then $S=]-1,+1[\times]-1,+1[$ after a translation. Define on $\mathcal{W}$ the family of functions $\left(\theta_{p, q}^{i}\right)_{p, q \in N}^{i=1, \ldots, 4}$ as follows :

$$
\theta_{p, q}^{i}(x, y, z)=\frac{1}{\pi \sqrt{p^{2}+q^{2}}} \vartheta_{p, q}^{i}(x, y) \sinh \left(\pi z \sqrt{p^{2}+q^{2}}\right)
$$

where $\left(\vartheta_{p, q}^{i}\right)_{p, q \in N}^{i=1, \ldots, 4}$ is the orthogonal basis of $L^{2}(S)$ defined as follows :

$$
\left\{\begin{array}{l}
\vartheta_{p, q}^{1}(x, y)=\cos (p \pi x) \cos (q \pi y) \\
\vartheta_{p, q}^{2}(x, y)=\cos (p \pi x) \sin (q \pi y) \\
\vartheta_{p, q}^{3}(x, y)=\sin (p \pi x) \cos (q \pi y) \\
\vartheta_{p, q}^{4}(x, y)=\sin (p \pi x) \sin (q \pi y)
\end{array}\right.
$$

Lemma 1 Let $\widetilde{\left[u_{\sigma}\right]}$ be the extension by zero of $\left[u_{\sigma}\right]$ to $S$. Then, for $p, q \in N$ and $i=1,2,3,4$ :

$$
R G_{[\phi, f]}\left(\theta_{p, q}^{i}\right)=\int_{S} \widetilde{\left[u_{\sigma}\right]} \cdot \vartheta_{p, q}^{i}
$$




\section{$\underline{\text { Remarks : }}$}

1. It is worthwhile to observe that $\widetilde{\left[u_{\sigma}\right]}$ can be defined as a function of $H^{\frac{1}{2}}(S)$ since $\left[u_{\sigma}\right] \in H_{00}^{\frac{1}{2}}(\sigma)$, and the extension by zero maps continuously $H_{00}^{\frac{1}{2}}(\sigma)$ onto $H^{\frac{1}{2}}(S)([17])$.

2. Equations (14) give in fact the Fourier coefficients of $\widetilde{\left[u_{\sigma}\right]}$ on the square $S$. By reconstructing its Fourier expansion, we can then determine its support.

Of course, we shall use a truncated Fourier expansion, the so called quadratic partial sum at order $n$ :

$$
\widetilde{\left[u_{\sigma}\right]_{n}}=\sum_{p, q=1}^{n} \sum_{i=1}^{4} R G_{[\phi, f]}\left(\theta_{p, q}^{i}\right) \cdot \vartheta_{p, q}^{i}
$$

As mentioned above, the support of $\widetilde{\left[u_{\sigma}\right]}$ is exactly the crack $\bar{\sigma}$. The following recalls this statement, proved in [7].

Proposition $3\left[u_{\sigma}\right]$ cannot take a non nill constant value on any open subset of positive measure of $\sigma$. As for the set $\left\{x \in \sigma ;\left[u_{\sigma}\right](x)=0\right\}$, it is either of nill measure, or equal to $\sigma$.

Remark : Actually, $\left\{x \in \sigma ;\left[u_{\sigma}\right](x)=0\right\}=\sigma$ means that the chosen flux $\phi$ is not identifying.

\section{The numerical semi-explicit algorithm.}

Following the above results, the algorithm appears clearly to split in two steps :

1. The first one, which is explicit, consists in locating - by using formulae (8) and (10) given in the former section - the plane (П) containing the cracks. This determination is exact, so far as the data are exact themselves.

2. The second one is the numerical computation, by use of formula (14), of the truncated Fourier expansion (15) of the solution jump $\left[u_{\sigma}\right]$.

The only numerical computations needed by the algorithm are those of the Fourier coefficients of $\widetilde{\left[u_{\sigma}\right]}$, which are obtained by computing integrals on the external boundary involving both the prescribed data $\phi$ and the measured data $f$ :

$$
R G_{[\phi, f]}\left(\theta_{p, q}^{i}\right)=\int_{S} \widetilde{\left[u_{\sigma}\right]} \cdot \vartheta_{p, q}^{i}=\int_{\partial \Omega} \phi \theta_{p, q}^{i}-\frac{\partial \theta_{p, q}^{i}}{\partial n} f
$$

In this paper, all the numerical trials have been run with synthetic data, i.e. with data generated by a finite element computation providing $f$ from the prescribed flux $\phi$.

It should be pointed out that we are interested here in approximating the support of this jump, rather than the jump itself. For this purpose, the truncated Fourier series $\widetilde{\left[u_{\sigma}\right]_{n}}$ is not expected to be satisfactory, since its support has no reason not to extend on the whole domain $S$. In order to provide an approximation of the cracks, we need then to define, for a given positive real number $\varepsilon$, and a given integer $n$, the following sets :

$$
\sigma_{\varepsilon}=\left\{x \in S ;\left|\left[u_{\sigma}\right](x)\right|>\varepsilon\right\}
$$

and

$$
\sigma_{n \varepsilon}=\left\{x \in S ;\left|\widetilde{\left[u_{\sigma}\right]_{n}}(x)\right|>\varepsilon\right\}
$$

The first one is expected to be an approximation of $\sigma$, and the second one an approximation of $\sigma_{\varepsilon}$. Let us denote by $d$ the Hausdorff distance in the plane (П). The following result then holds : $\mathrm{RR} \mathrm{n}^{\circ} 3063$ 


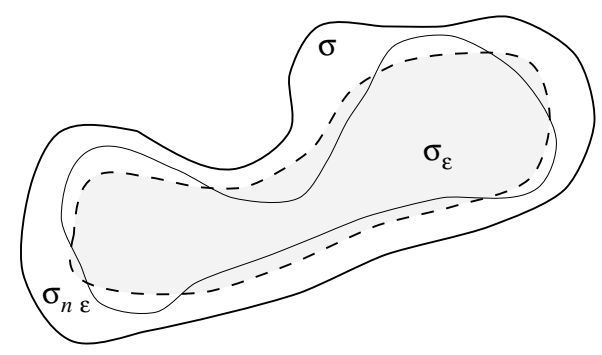

Figure 2: Approximations of the crack

Lemma 2 For a prescribed real positive number $\varepsilon$, we have:

$$
\lim _{n \rightarrow \infty} d\left(\sigma_{n \varepsilon} ; \sigma_{\varepsilon}\right)=0
$$

and furthermore :

$$
\lim _{\varepsilon \rightarrow 0} \lim _{n \rightarrow \infty} d\left(\sigma_{n \varepsilon} ; \sigma\right)=0
$$

Proof : For sake of simplicity, we shall denote by $g$ the unknown function $\widetilde{\left[u_{\sigma}\right]}$ that we are seeking to approximate, and by $g_{n}$ its truncated Fourier expansion, at order $\mathrm{n}$. Then ;

$$
\sigma_{\varepsilon}=\{x \in S ;|g(x)|>\varepsilon\}
$$

and

$$
\sigma_{n \varepsilon}=\left\{x \in S ;\left|g_{n}(x)\right|>\varepsilon\right\}
$$

The sets $\sigma_{\varepsilon}$ are strictly decreasing with respect to $\varepsilon$, i.e. :

$$
\sigma_{\varepsilon} \subset \sigma_{\eta} \subset \sigma \text { if } \varepsilon>\eta
$$

We are going to prove that, for a given $\varepsilon$, there exists some integer $N$ such that :

$$
n \geq N \Rightarrow \sigma_{\frac{3 \varepsilon}{2}} \subseteq \sigma_{n \varepsilon} \subseteq \sigma_{\frac{\varepsilon}{2}} \subseteq \sigma
$$

To this end, we shall need the Grisvard's regularity results for solutions of elliptic problems in singular domains $([17]): u_{\sigma} \in H^{\frac{3}{2}-\gamma}\left(\Omega_{\sigma}\right)$, and therefore that $\left.\left[u_{\sigma}\right]\right|_{\sigma} \in H_{00}^{\frac{1}{2}} \cap H^{1-\gamma}(\sigma)$ for any positive real number $\gamma$. Then $g \in H^{1-\gamma}(S)$, and it can be locally (i.e. near the crack boundary $\partial \sigma$ ) expanded into a regular part $g^{R} \in H^{\frac{3}{2}}(S)$, and a "singular part" $g^{S}$ which behaves like $\rho^{\frac{1}{2}}, \rho$ being the distance to the crack boundary $\partial \sigma$. It turns out then that $g$ is a continuous function, and even an absolutely continuous one on $\partial \sigma$, so that the following holds :

$$
\lim _{\varepsilon \rightarrow 0} d\left(\sigma_{\varepsilon} ; \sigma\right)=\lim _{\varepsilon \rightarrow 0} \mu(\{x \in S ; 0<g(x) \leq \varepsilon\})=0
$$

$\mu$ being the Lebesgue measure in (П).

On another hand, we need to prove that the Fourier quadratic partial sum $g_{n}$ is uniformly convergent to $g$. This is done by using a result due to Zhizhiashvili (1971 and 1973), reported by Alimov et al [5], which is a generalisation of the Dini-Lipshitz uniform convergence of Fourier series theorem from the $1 \mathrm{D}$ to the multi-dimensional case. Namely, the statement is that, if the modulus of continuity $\omega(\delta ; g):=\max _{|x-y|<\delta}|g(x)-g(y)|$ has the following behaviour :

$$
\omega(\delta ; g)=o(1)\left(\log \frac{1}{\delta}\right)^{-2} \text { for } \delta \rightarrow 0 \text { (in the 2D-case) }
$$


then the quadratic partial sums converge uniformly to $g$ on $S$. Let us check the behaviour of the continuity modulus. According to its regularity, $g$ is a $\mathcal{C}^{\frac{1}{2}-\gamma}$ function, for any $\gamma>0$ [1], and then :

$$
|g(x)-g(y)| \leq c|x-y|^{\frac{1}{2}-\gamma}
$$

such that $\omega(\delta ; g)=O\left(\delta^{\frac{1}{2}-\gamma}\right)$, which insures (23) and, according to Zhizhiashvili, the uniform convergence of $g_{n}$ to $g$. For any given $\varepsilon$, there exists then some integer $N(\varepsilon)$ such that :

$$
n \geq N(\varepsilon) \Rightarrow \max _{x \in S}\left|g_{n}(x)-g(x)\right| \leq \frac{\varepsilon}{2}
$$

Let now $x \in \sigma_{n \varepsilon}$. Then :

$$
|g(x)| \geq\left|g_{n}(x)\right|-\left|g(x)-g_{n}(x)\right|>\varepsilon-\frac{\varepsilon}{2}=\frac{\varepsilon}{2}
$$

which proves that for $n \geq N(\varepsilon), \sigma_{n \varepsilon} \subseteq \sigma_{\frac{\varepsilon}{2}}$. By the same kind of argument, $\sigma_{\frac{3 \varepsilon}{2}} \subseteq \sigma_{n \varepsilon}$ and (21) is proved. Since we also have $\sigma_{\frac{3 \varepsilon}{2}} \subset \sigma_{\varepsilon} \subset \sigma_{\frac{\varepsilon}{2}}$, we can derive that $d\left(\sigma_{n \varepsilon} ; \sigma_{\varepsilon}\right) \leq d\left(\sigma_{\frac{3 \varepsilon}{2}} ; \sigma_{\frac{\varepsilon}{2}}\right)$, which - by using (22) - proves (18).

This result means that for a fixed $\varepsilon, \lim _{n \rightarrow \infty} \sigma_{n \varepsilon}=\sigma_{\varepsilon} . d$ being continuous, we immediatly get :

$$
\lim _{n \rightarrow \infty} d\left(\sigma_{n \varepsilon} ; \sigma\right)=d\left(\sigma_{\varepsilon} ; \sigma\right)
$$

and finally :

$$
\lim _{\varepsilon \rightarrow 0} \lim _{n \rightarrow \infty} d\left(\sigma_{n \varepsilon} ; \sigma\right)=\lim _{\varepsilon \rightarrow 0} d\left(\sigma_{\varepsilon} ; \sigma\right)=0
$$

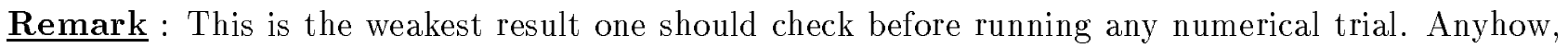
it shows - since $\lim _{n \rightarrow \infty} \lim _{\varepsilon \rightarrow 0} \sigma_{n \varepsilon}=S$, while $\lim _{\varepsilon \rightarrow 0} \lim _{n \rightarrow \infty} \sigma_{n \varepsilon}=\sigma$ - that the numerical trials have to be performed at a fixed "tolerance number" $\varepsilon$, and that $\sigma_{n \varepsilon}$ is the right candidate to approximate $\sigma$. By the forthcoming error estimates, we establish more precisely this statement.

Lemma 3 Under the assumption that the "stress intensity factor" does not vanish on $\partial \sigma$, there exists some constant $c$, and some real positive number $\varepsilon_{0}$ depending on $\Omega_{\sigma}$ and $u_{\sigma}$, such that for any $\varepsilon \leq \varepsilon_{0}$, we have :

$$
d\left(\sigma_{\varepsilon} ; \sigma\right) \leq c . \varepsilon^{2}
$$

Proof : Using again Grisvard's regularity results, one has :

$$
g(x)=g^{R}(x)+\rho^{\frac{1}{2}} \varphi(s)
$$

where $\rho$ is the distance to the boundary $\partial \sigma, \varphi(s)$ being the so-called stress intensity factor by analogy with the elasticity problem. Namely $\varphi$ is some regular function of the curvilign abcissa of $\partial \sigma$, and $g^{R}$ a regular function defined on $\sigma\left(g^{R} \in H^{2-\gamma}(\sigma) \cap H_{0}^{1}(\sigma)\right)$. Let $x$ be a point of $\partial \sigma_{\varepsilon}$, and $x_{0}$ a point of $\partial \sigma$ such that :

$$
\operatorname{dist}(x ; \partial \sigma)=\operatorname{dist}\left(x ; x_{0}\right)=\left|x-x_{0}\right|
$$

Then, we have, since $\rho(x)=\left|x-x_{0}\right|$ and $g^{R}\left(x_{0}\right)=0$ :

$$
g(x)=g^{R}(x)-g^{R}\left(x_{0}\right)+\rho^{\frac{1}{2}} \varphi\left(s_{0}\right)
$$

For any $x \in \partial \sigma_{\varepsilon}$, it comes then, since $|g(x)|=\varepsilon$ :

$\operatorname{RR~} n^{\circ} 3063$

$$
\varepsilon \geq \rho^{\frac{1}{2}}\left|\varphi\left(s_{0}\right)\right|-\left|g^{R}(x)-g^{R}\left(x_{0}\right)\right|
$$


Since $g^{R} \in H^{2-\gamma}(\sigma)$, there is some constant $c_{2}$ depending on $g$ and $\sigma$ such that :

$$
\left|g^{R}(x)-g^{R}\left(x_{0}\right)\right| \leq c_{2}\left|x-x_{0}\right|
$$

By the assumption that the "stress intensity factor" $\varphi$ does not vanish on $\partial \sigma$, and thus that $\min _{s \in \partial \sigma}|\varphi(s)| \geq$ $\beta>0$, we get then :

$$
\varepsilon \geq\left(\beta-c_{2} \rho^{\frac{1}{2}}\right) \rho^{\frac{1}{2}} \quad \forall x \in \partial \sigma_{\varepsilon}
$$

The mapping $\varepsilon \longmapsto \max _{x \in \partial \sigma_{\varepsilon}} \rho(x)$ is a positive increasing function of $\varepsilon$. Then :

$$
\lim _{\varepsilon \rightarrow 0} \max _{x \in \partial \sigma_{\varepsilon}} \rho(x)=0
$$

because if not, this limit would be some positive real number $\mu$, and one could exhibit a sequence $x_{m} \in \partial \sigma_{\frac{1}{m}}$ such that $\lim _{m \rightarrow \infty} \rho\left(x_{m}\right)=\mu$. From $\left(x_{m}\right)_{m \geq 1}$, we could then extract a convergent subsequence $x_{m_{k}}$, with limit $x^{*} \in \bar{\sigma}$, and $x^{*} \in \partial \sigma$ since :

$$
g\left(x^{*}\right)=\lim _{k \rightarrow \infty} g\left(x_{m_{k}}\right)=\lim _{k \rightarrow \infty}\left(\frac{1}{m_{k}}\right)=0
$$

This cannot happen because $\lim _{k \rightarrow \infty} \operatorname{dist}\left(x_{m_{k}} ; \partial \sigma\right)=\lim _{k \rightarrow \infty} \rho\left(x_{m_{k}}\right)=\mu \neq 0$.

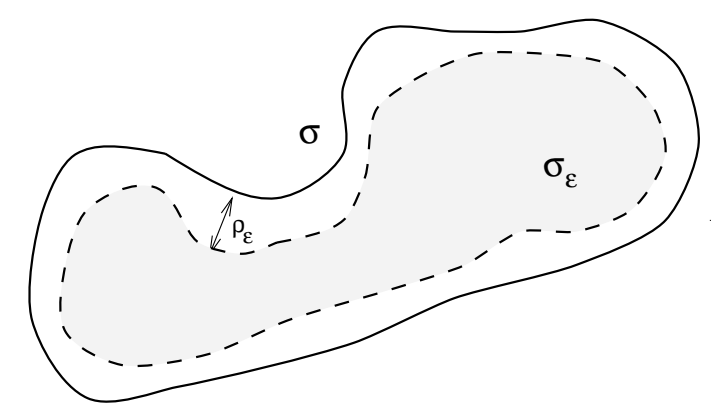

Figure 3: Distance between the crack and its approximation

It follows from (27) and (28) that we can find two real positive numbers $\varepsilon_{0}$ and $\tilde{\beta}$ such that, for any $\varepsilon \leq \varepsilon_{0}$, we have :

$$
\beta-c_{2} \rho^{\frac{1}{2}} \geq \tilde{\beta}>0 \text { for all } x \in \partial \sigma_{\varepsilon}
$$

Let us now define $\rho_{\varepsilon}=\max _{x \in \partial \sigma_{\varepsilon}} \min _{y \in \partial \sigma} \operatorname{dist}(x ; y)$. Using $(27)$, it comes then :

$$
\varepsilon \geq \tilde{\beta} \rho_{\varepsilon}^{\frac{1}{2}}
$$

On another hand, any point of $\partial \sigma_{\varepsilon}$ is at a distance less than $\rho_{\varepsilon}$ from $\partial \sigma$, and consequently, since $\sigma_{\varepsilon} \subset \sigma$, no point of $\sigma \backslash \sigma_{\varepsilon}$ is at a distance from $\partial \sigma$ greater than $\rho_{\varepsilon}$. Thus, $d\left(\sigma_{\varepsilon} ; \sigma\right)=\mu\left(\sigma \backslash \sigma_{\varepsilon}\right)$ is bounded by the measure of a strip of length $\lambda(\partial \sigma)$, which is the "measure" of $\partial \sigma$, and of width $\rho_{\varepsilon}$ :

$$
d\left(\sigma_{\varepsilon} ; \sigma\right) \leq \rho_{\varepsilon} \lambda(\partial \sigma)
$$

which leads to $\varepsilon \geq \tilde{\gamma} d\left(\sigma_{\varepsilon} ; \sigma\right)^{\frac{1}{2}}$, with $\tilde{\gamma}=\tilde{\beta} \lambda(\partial \sigma)$. This proves (25). 


\section{Remarks :}

1. Estimate (25) is strongly based on the knowledge we have of the behaviour of the singular part of the solution. Actually, the power of $\varepsilon$ is the inverse of the power of the local behaviour (near the crack tip) of the solution.

2. All the above proofs, given in the case of a single crack, extend easily to the case of multiple co-planar cracks, by reasoning on each connected component of $\sigma$.

3. In the $2 \mathrm{D}$ case, all the proofs work as well, and are even simpler : the Hausdorff distance reduces then to the sum of the distances between the left and right crack tips while $\rho_{\varepsilon}$ is the maximum value of these two distances.

We are now able to give the final estimate in the following theorem.

Theorem 1 Under the assumption that the "stress intensity factor" does not vanish on $\partial \sigma$, and given any positive number $\delta$, there exists two positive constants $c$ and $\tilde{c}$, and some real positive number $\varepsilon_{0}$, such that for $\varepsilon \leq \varepsilon_{0}$, and $n>\tilde{c} \varepsilon^{-(2+\delta)}$, we have :

$$
d\left(\sigma_{n \varepsilon} ; \sigma\right) \leq c \cdot \varepsilon^{2}
$$

Proof : We shall use the following characterization of the Sobolev space $H^{s}(S)[18$, Kress] :

$$
H^{s}(S)=\left\{g \in L^{2}(S) ; \sum_{p, q=1}^{\infty} \sum_{i=1}^{4}\left(1+p^{2}+q^{2}\right)^{s}\left|a_{p q}^{i}(g)\right|^{2}<\infty\right\}
$$

where $a_{p q}^{i}(g)$ are the Fourier coefficients of $g$, the space $L^{2}(S)$ being the set of functions $g$ verifying $\sum_{p, q=1}^{\infty} \sum_{i=1}^{4}\left|a_{p q}^{i}(g)\right|^{2}<\infty$. Given a positive number $\gamma<1$, which relationship to the given $\delta$ will be precised later on, we have already pointed out that $g \in H^{s}(S)$ for $s=1-\gamma$. We have :

$$
\left(g-g_{n}\right)(x)=\sum_{p, q>n} \sum_{i=1}^{4} a_{p q}^{i}(g) \vartheta_{p q}^{i}(x)
$$

which can also be written :

$$
\left(g-g_{n}\right)(x)=\sum_{p, q>n} \sum_{i=1}^{4}\left(1+p^{2}+q^{2}\right)^{-\frac{s}{2}}\left(1+p^{2}+q^{2}\right)^{\frac{s}{2}} a_{p q}^{i}(g) \vartheta_{p q}^{i}(x)
$$

Using the Cauchy-Schwarz inequality, we get :

$$
\max _{x \in S}\left|\left(g-g_{n}\right)(x)\right| \leq\left\{\sum_{p, q>n}\left(1+p^{2}+q^{2}\right)^{-s}\right\}^{\frac{1}{2}}\left\{\sum_{p, q>n} \sum_{i=1}^{4}\left(1+p^{2}+q^{2}\right)^{s}\left|a_{p q}^{i}(g)\right|^{2}\right\}^{\frac{1}{2}}
$$

Since $g \in H^{s}$, the second term between brackets is converging to 0 , and is then bounded by some $\kappa>0$. Thus, chosing any real positive number $s^{\prime}$ smaller than $s$, we derive :

$\operatorname{RR~} n^{\circ} 3063$

$$
\max _{x \in S}\left|\left(g-g_{n}\right)(x)\right| \leq \kappa\left\{\sum_{p, q>n}\left(1+p^{2}+q^{2}\right)^{-s^{\prime}}\left(1+p^{2}+q^{2}\right)^{-\left(s-s^{\prime}\right)}\right\}^{\frac{1}{2}}
$$


and therefore :

$$
\max _{x \in S}\left|\left(g-g_{n}\right)(x)\right| \leq \frac{\kappa}{\left(1+2 n^{2}\right)^{\frac{s^{\prime}}{2}}}\left\{\sum_{p, q>n}\left(1+p^{2}+q^{2}\right)^{-\left(s-s^{\prime}\right)}\right\}^{\frac{1}{2}}
$$

Now, any choice of $s^{\prime}$ such that $s-s^{\prime}>\frac{1}{2}$ would insure the convergence of the series between brackets, which is therefore bounded. Such a choice is realized by taking $s^{\prime}=\frac{1-2 \gamma^{\prime}}{2}$, with $\frac{1}{2}>\gamma^{\prime}>\gamma$. We obtain then :

$$
\left|\left(g-g_{n}\right)(x)\right| \leq \frac{\tilde{\kappa}}{n^{\frac{1}{2}-\gamma^{\prime}}}
$$

Thus, in order to get $\left|\left(g-g_{n}\right)(x)\right| \leq \frac{\varepsilon}{2}$, it is sufficient to choose $N(\varepsilon) \geq \tilde{c} \varepsilon^{-\frac{2}{1-2 \gamma^{\prime}}}$, where $\tilde{c}=(2 \tilde{\kappa})^{\frac{2}{1-2 \gamma^{\prime}}}$. Let us then set $\gamma^{\prime}=\frac{\delta}{2(2+\delta)}$, which fulfills the required condition $0<\gamma^{\prime}<\frac{1}{2}$, and chose for $\gamma$ any real positive number smaller than $\gamma^{\prime}$. The condition on $N(\varepsilon)$ becomes then $N(\varepsilon) \geq \tilde{c} \varepsilon^{-(2+\delta)}$.

Now, by lemma 3 , we know the existence of two positive numbers $\varepsilon_{0}$ and $c$ such that $\varepsilon \leq \varepsilon_{0} \Rightarrow d\left(\sigma_{\frac{3 \varepsilon}{2}} ; \sigma\right) \leq$ $c \varepsilon^{2}$. Chosing $n>\tilde{c} \varepsilon^{-(2+\delta)}$ insures then (24), which is :

$$
\max _{x \in S}\left|\left(g-g_{n}\right)(x)\right| \leq \frac{\varepsilon}{2}
$$

and therefore, as in the proof of lemma $2, \sigma_{\frac{3 \varepsilon}{2}} \subseteq \sigma_{n \varepsilon} \subseteq \sigma_{\frac{\varepsilon}{2}}$. Then :

$$
d\left(\sigma_{n \varepsilon} ; \sigma\right) \leq d\left(\sigma_{\frac{3 \varepsilon}{2}} ; \sigma\right) \leq c \varepsilon^{2}
$$

which is the desired estimate.

\section{Numerical results.}

The numerical study has been carried for $2 \mathrm{D}$ and $3 \mathrm{D}$ cases, but according to the computer facilities available, the algorithm behaviours have been outlined for $2 \mathrm{D}$ situations, some $3 \mathrm{D}$ cases been run afterwards to make sure that the conclusions extend. Furthermore, the 2D numerical trials have been mostly carried for a square domain $\Omega$, which is not actually a limitation in our case, since the first step of the algorithm is explicit and is hence not influenced by the domain's shape, and that its second step begins by immersing the domain in some "square big box" before computing the Fourier series. Anyhow, some special geometries have also been achieved to complete the study.

\subsection{Location of the crack's line}

According to section 2, the location of the crack's line is achieved by computing the three boundary integrals involved in the formulae (8) and (10) giving the two components of the normal vector, and the constant of the line's equation. Any flux $\phi$ satisfying the identifiability condition $\int_{\sigma}\left[u_{\sigma}\right] \neq 0$ can be used in the identification process, but it is expected that some fluxes behave better than others. Referring to Friedman and Vogelius ([16]), we know that non identifying fluxes exist, for which the crack is immerged in some field line of the solution. 
As mentioned above, all our trials are based on synthetic data, obtained via a finite element direct computation using the INRIA's Modulef library. The boundary integrals have then been computed by using a Simpson's rule on the finite element mesh. Several fluxes have been tested, to determine the line supporting a crack $\sigma$ with endpoints $(0.5,0$. $)$ and $(0.8,0$.), within the square domain $\Omega=$ ]-1,1[ $\times]-1,1\left[\right.$. The constant is determined with less that a $10^{-4}$ error in all the cases, while the angle (i.e. the normal vector) is determined with a better accuracy. In figure 4, we can notice that - with 20 basis functions - the reconstitution of the crack depends on the fluxes used to perform the algorithm,
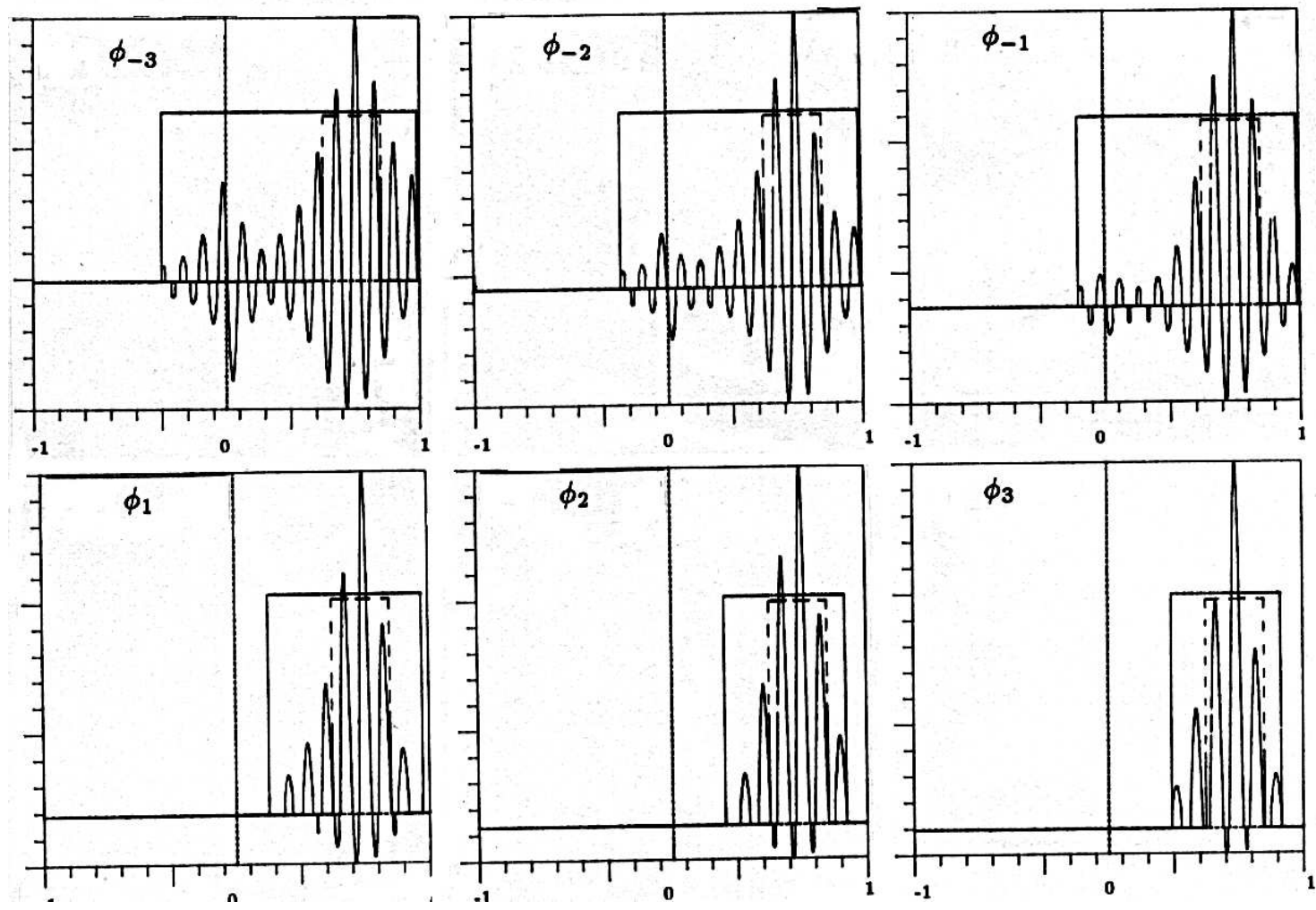

Figure 4: Reconstitution of a crack using six different fluxes (20 basis functions, $5 \%$ tolerance number)

the "worst" approximation being obtained in the case where the level lines are crossing almost continuously the crack (and thus $\left[u_{\sigma}\right]$ is almost vanishing on $\sigma$ ). Figure 5 illustrates this statement. Comparing the level lines for the "worst" flux $\left(\phi_{-3}\right)$ and the "best" one $\left(\phi_{3}\right)$, we notice - for this latter's - strong discontinuities across the crack, and also high gradients near the crack tips, which denote the presence of singularities due to non vanishing stress intensity factors. Even this assumption is not necessary to insure the identifiability, it was needed to prove a Lipschitz-stability result for $2 \mathrm{D}$ emerging cracks [9], and it was - in the present paper - essential to derive the error estimate (25) of lemma 3 , and thus to derive the error estimate (29) in theorem 1.

\subsection{Complete identification and convergence}

The crack's line being identified, we know are first going to look at the effects of the truncation of the Fourier expansion on the accuracy on the reconstructed jump $\left[u_{\sigma}\right]$, before being interested in its support. Figure 6 gives the "real" jump (obtained by the finite element computation), and the reconstructed jumps obtained by truncated Fourier series involving 20,30, 50, and 70 basis functions. Two observations can be pointed out at this stage :

1. The crack seems to be detected even at the lowest values of $n$; RR n ${ }^{\circ} 3063$ 


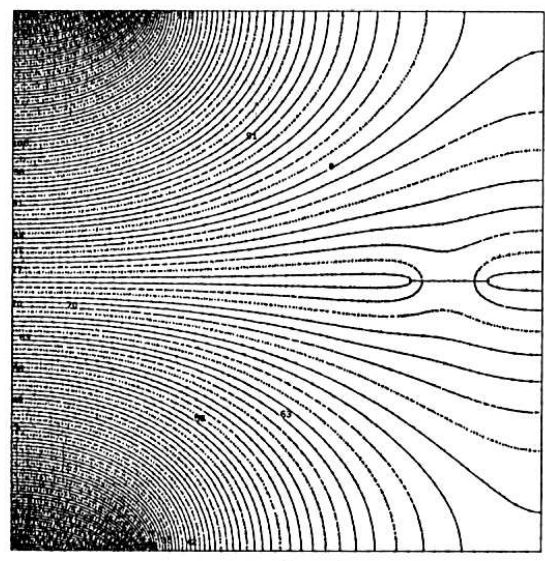

$a:$ Flux $\phi_{-3}$

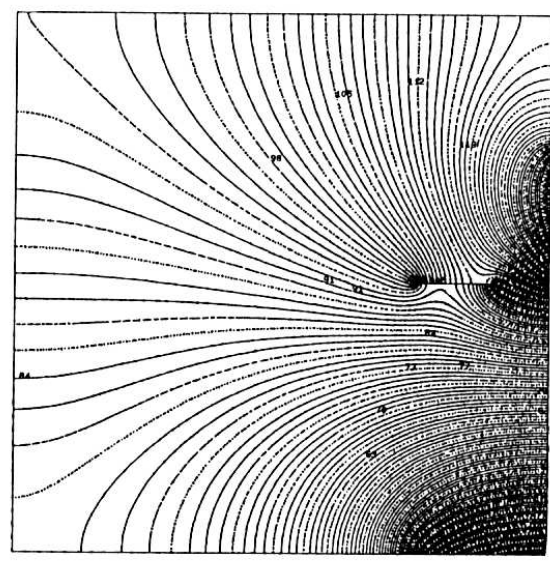

$b:$ Flux $\phi_{3}$

Figure 5: Level lines for the worst and the best flux (70 basis functions, $5 \%$ tolerance)

2. The reconstructed jump $\left.\widetilde{\left[u_{\sigma}\right]}\right|_{n}$ is somewhat oscillating, and cannot hence provide an acceptable approximation of the jump itself.
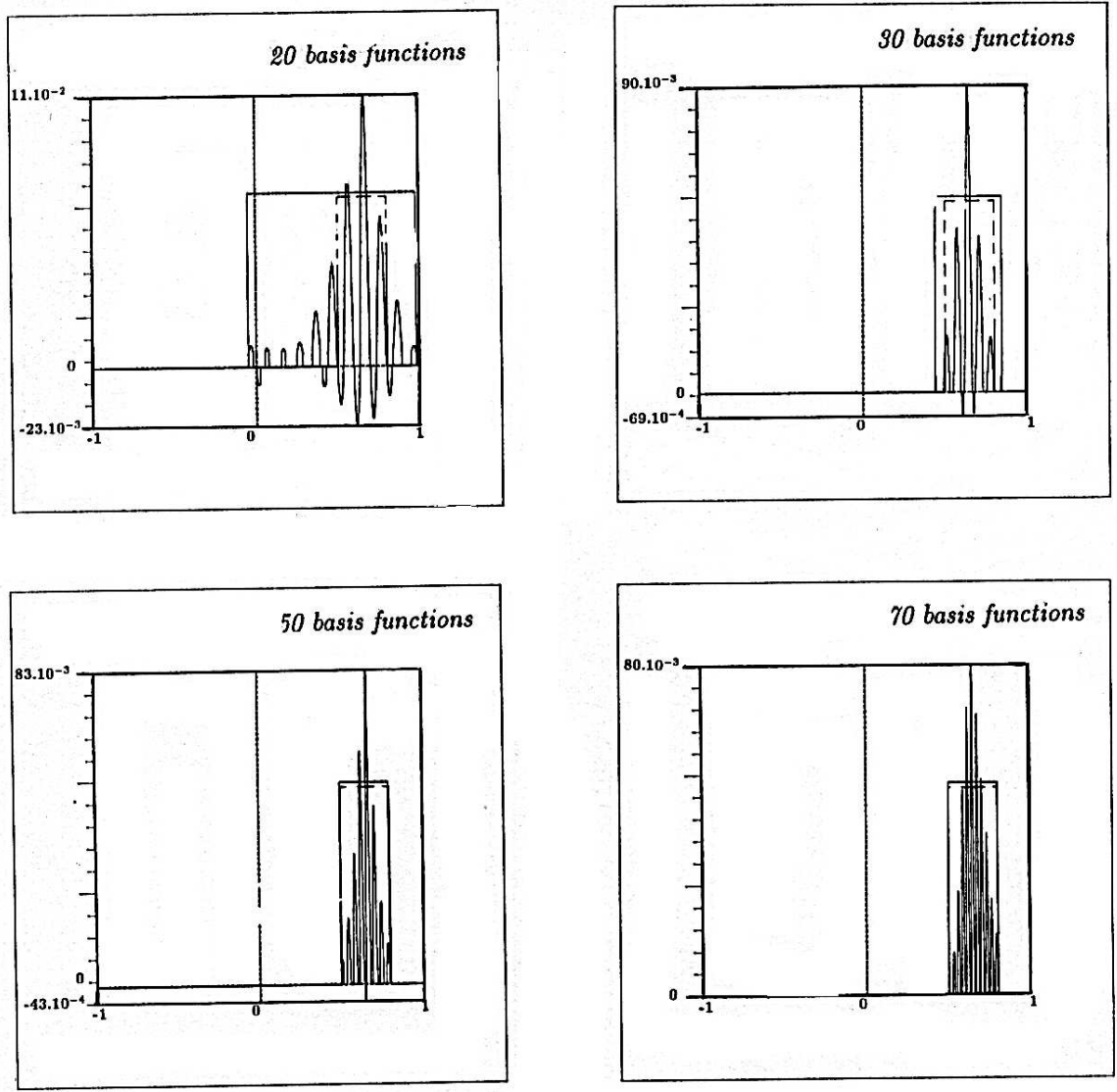

Figure 6: The reconstituted jumps and cracks using 20, 30, 50 and 70 basis functions ( $5 \%$ tolerance)

A smoothing procedure would probably improve the approximation of the jump, but it would in the same time spread out its support, which is the very information we are seeking to catch. As announced 
in section (3), we shall keep the jump as computed, and use for the approximation of its support the following set :

$$
\sigma_{n \varepsilon}=\left\{x \in S ;\left|\widetilde{\left[u_{\sigma}\right]}\right|_{n}(x) \mid>\varepsilon\right\}
$$

for a prescribed $\varepsilon$ that we shall refer at as the tolerance number.

Given a value of $n$ (which is a computational cost we are willing to pay), we should choose - according to theorem 1 - an $\varepsilon$ of order $n^{-\frac{1-\delta^{\prime}}{2}}$ - for any $\delta^{\prime}>0$ - so that we obtain an error $d\left(\sigma_{n \varepsilon} ; \sigma\right)$ of order $n^{-1+\delta^{\prime}}$. The behaviour of the errors, as shown in figure 7 , turns out however to be much better than expected.
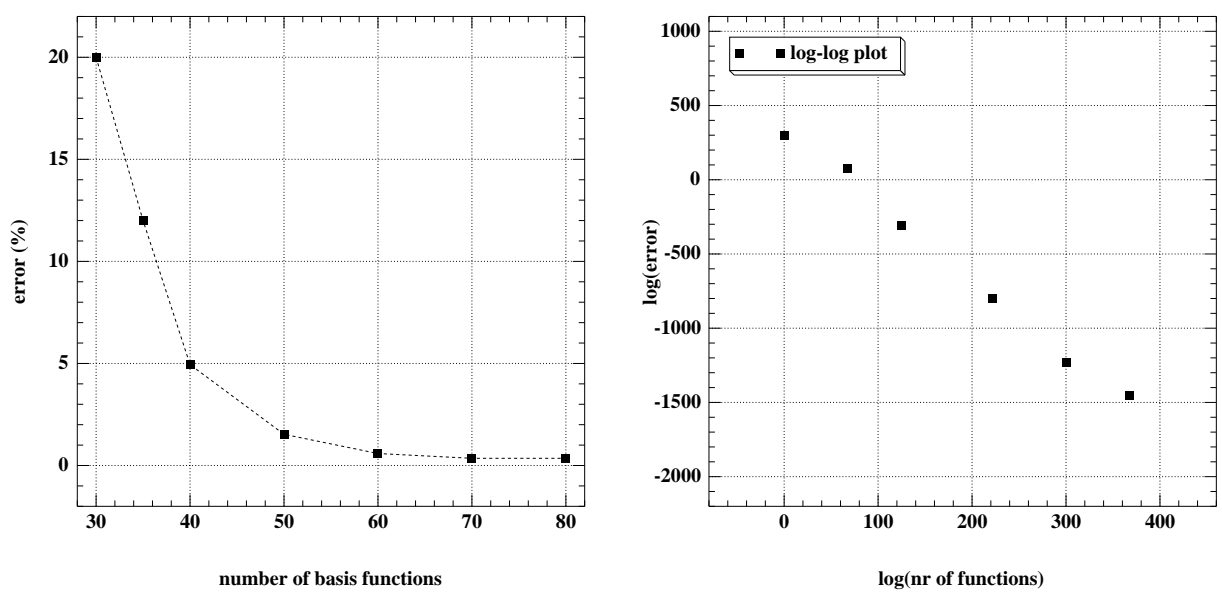

Figure 7: Error vs. number of basis functions used

As a matter of fact, the relationship between $n$ and $\varepsilon$, used to establish the error estimate (29), was based on the uniform convergence of the Fourier series, which is slow, and this could be a too strong requirement : observing the reconstituted cracks of figure 6 , one can notice that the convergence of $\sigma_{n \varepsilon}$ to $\sigma$ is achieved far ahead the uniform convergence of $g_{n}$ to $g$.
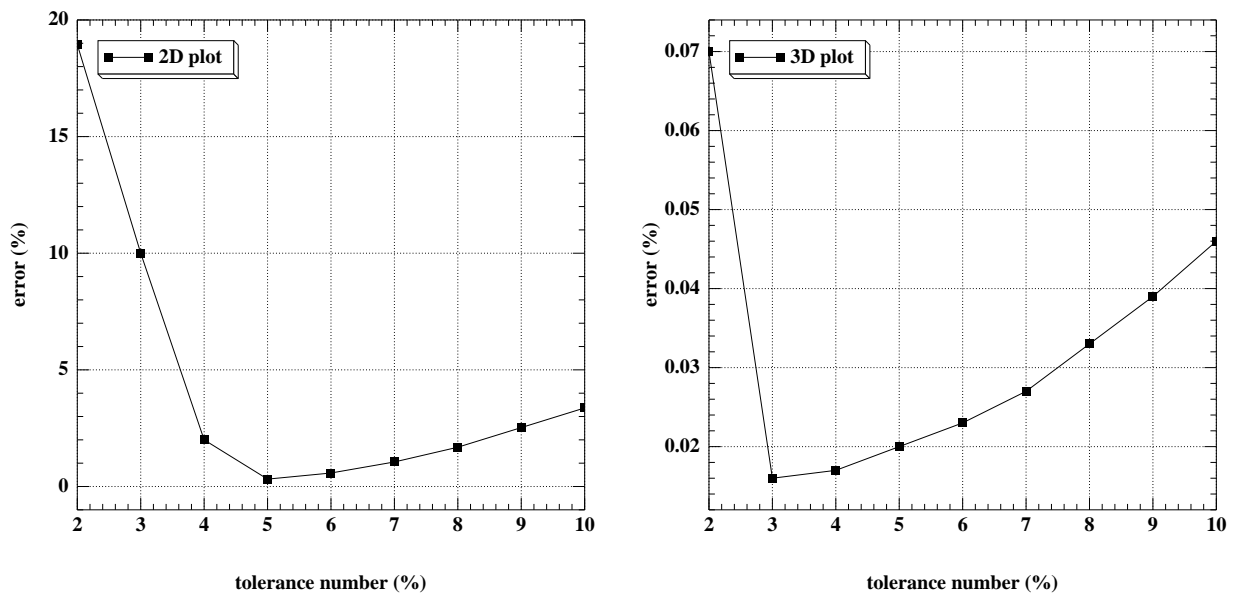

Figure 8: Error vs. tolerance number

According to these observations, all the trials reported from now on have been run with 70 basis functions in $2 \mathrm{D}$, and $70 \times 70$ functions in $3 \mathrm{D}$, which are the numbers it is not worthwile to go beyond $\operatorname{RR~}{ }^{\circ} 3063$ 
since they achieve the convergence of the cracks with an error (less than $0.4 \%$ ) which seems to be the lowest we can reach. The related tolerance numbers $(5 \%$ in $2 \mathrm{D}$, and $3 \%$ in $3 \mathrm{D})$ have been chosen according to the curves of figure 8 .

\subsection{Noisy data}

Running an identification algorithm based on measurements, one has to remind that measured data are subject to noise, the effects of which have to be studied. In our case, the data are synthetic, i.e. obtained by a finite element computation subject to errors, and they are hence already noisy. To the computational noise, we have added a random noise, generated by a Fortran routine, and run numerical trials for several ratios (with respect to the maximum value of the "measured" data) of this additinal noise.

Figure 9 gives the result of the complete identification algorithm for four ratios $(5,7,10$ and $15 \%)$. Up to $7 \%$ of additional noise, the results remain satisfactory. At $10 \%$ of additional noise, a parasite crack appears in the middle of the crack line. At $15 \%$ of additional noise, the crack is completely lost.
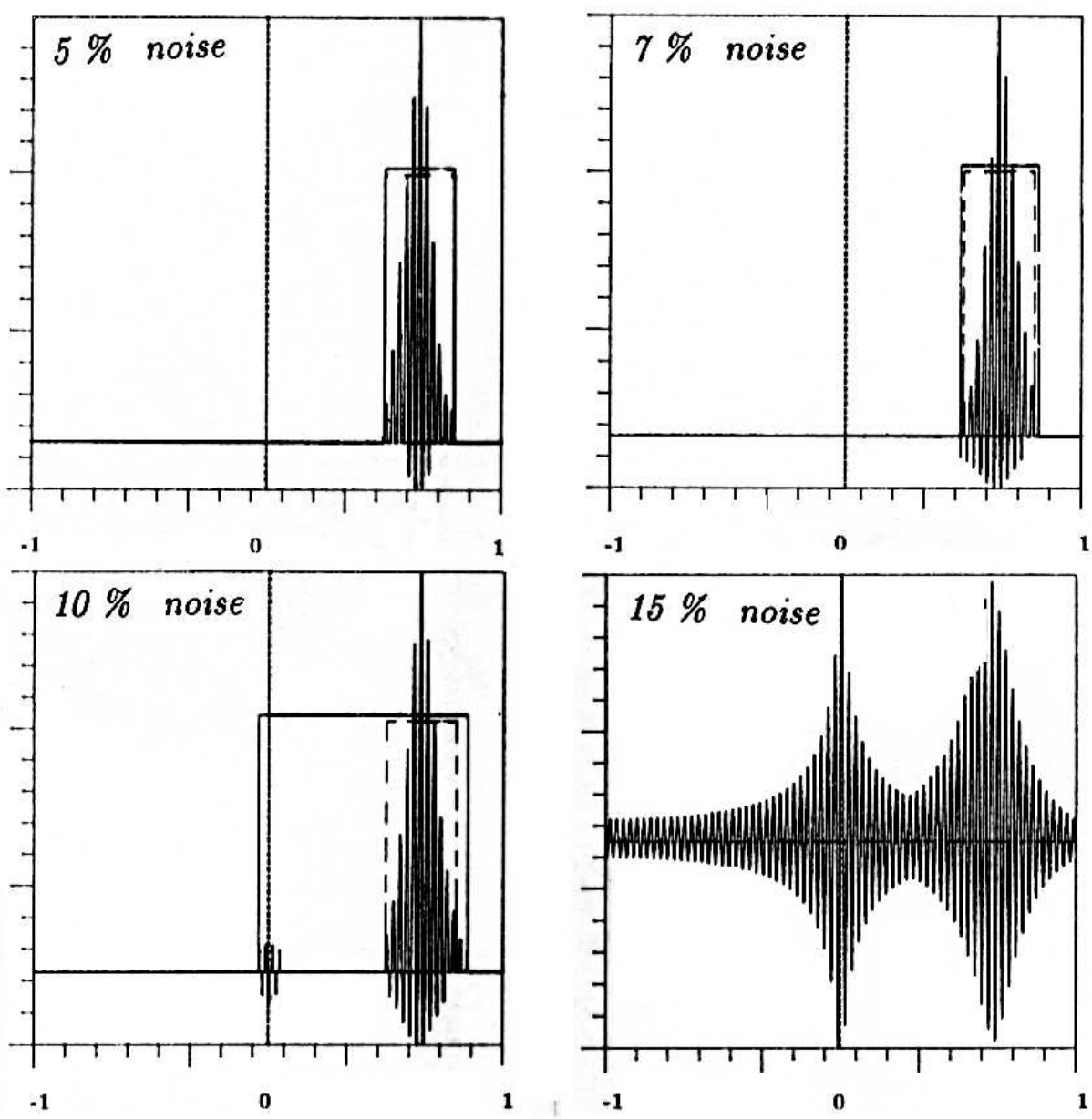

Figure 9: Behaviour of the algorithm with respect to the noise level

Actually, effects of the noise have been recorded even in the explicit part of the algorithm, namely in the determination of the crack's line, since this part involves as well the noisy data for the computation of the boundary integrals. The line is correctly identified up to $10 \%$ of additional noise, and it is lost at $20 \%$. At $15 \%$, the line is still correctly recovered, but the crack itself is lost [10, Bannour]. 


\subsection{Effects of the crack location}

No assumption on the location of the cracks is needed, from a theoretical viewpoint, by the algorithm to recover them. It is interesting therefore to verify, in a few extreme situations, how general the algorithm is. A crack located near the corner, an emerging crack (at an unknown boundary point), and a multiple crack were the three situations chosen for this test, the results of which are summarized in figure 10.
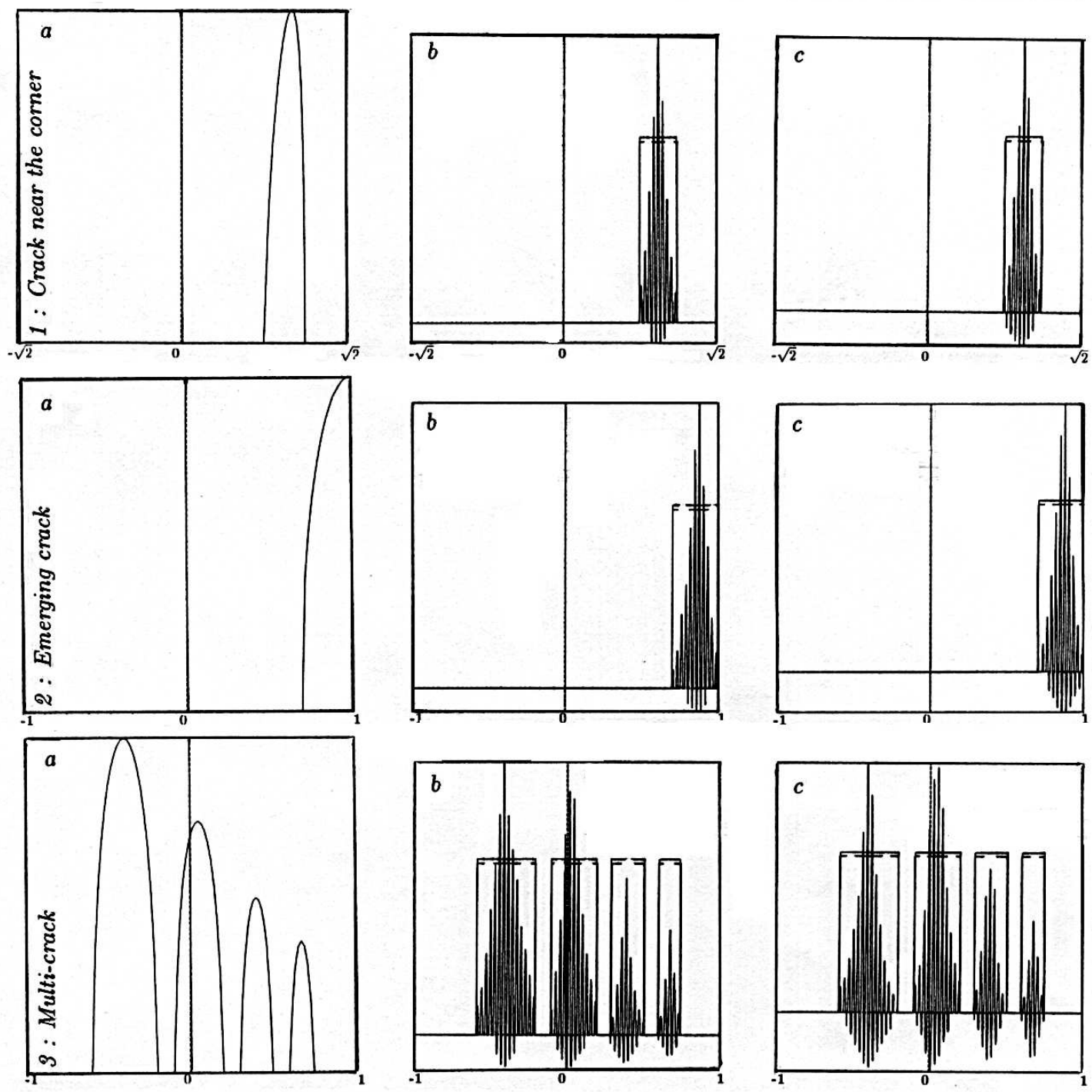

Figure 10: Recovery of a crack in some extreme situations : a-real jump ; b-reconstituted jump ; c-reconstituted crack

\subsection{Further trials and comments}

As announced in the beginning of this section, some "special" geometries have also been tested to verify that the process is not shape-sensitive, and 3D situations have also been studied. An exhaustive presentation of all these trials can be found in ([10, Bannour $]$ ). Figure 11 shows that the process is not shape-sensitive.

$\mathrm{RR} \mathrm{n}^{\circ} 3063$ 

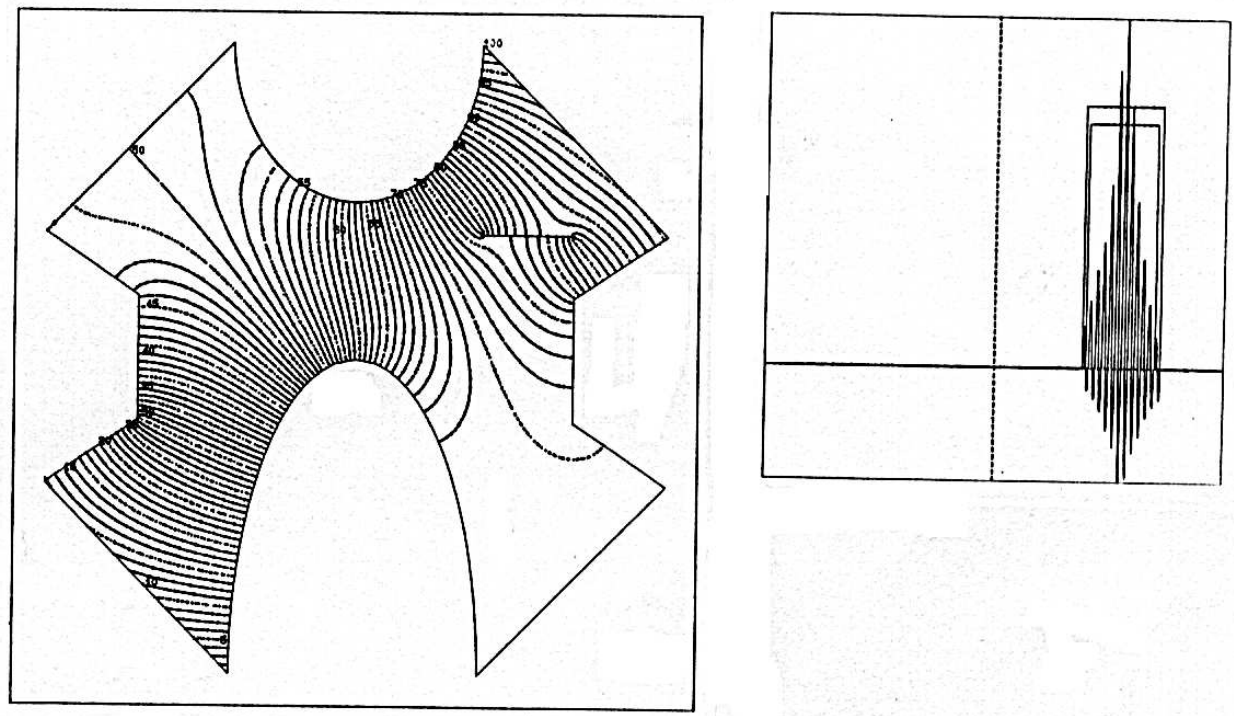

Figure 11: Recovery of cracks in the case of a non square domain

As expected, the 3D situations require much more computational time to run than the $2 \mathrm{D}$ ones : the number of Fourier basis functions needed is squared, and the integral computations on the boundary are $2 \mathrm{D}$ instead of being $1 \mathrm{D}$, so that the number of numerical integration is also squared, at a presribed rate of accuracy. Figure 12 gives the reconstitution of the jump through a square-shaped crack with respect to the number of harmonics $(30 \times 30,50 \times 50$, and $70 \times 70)$.
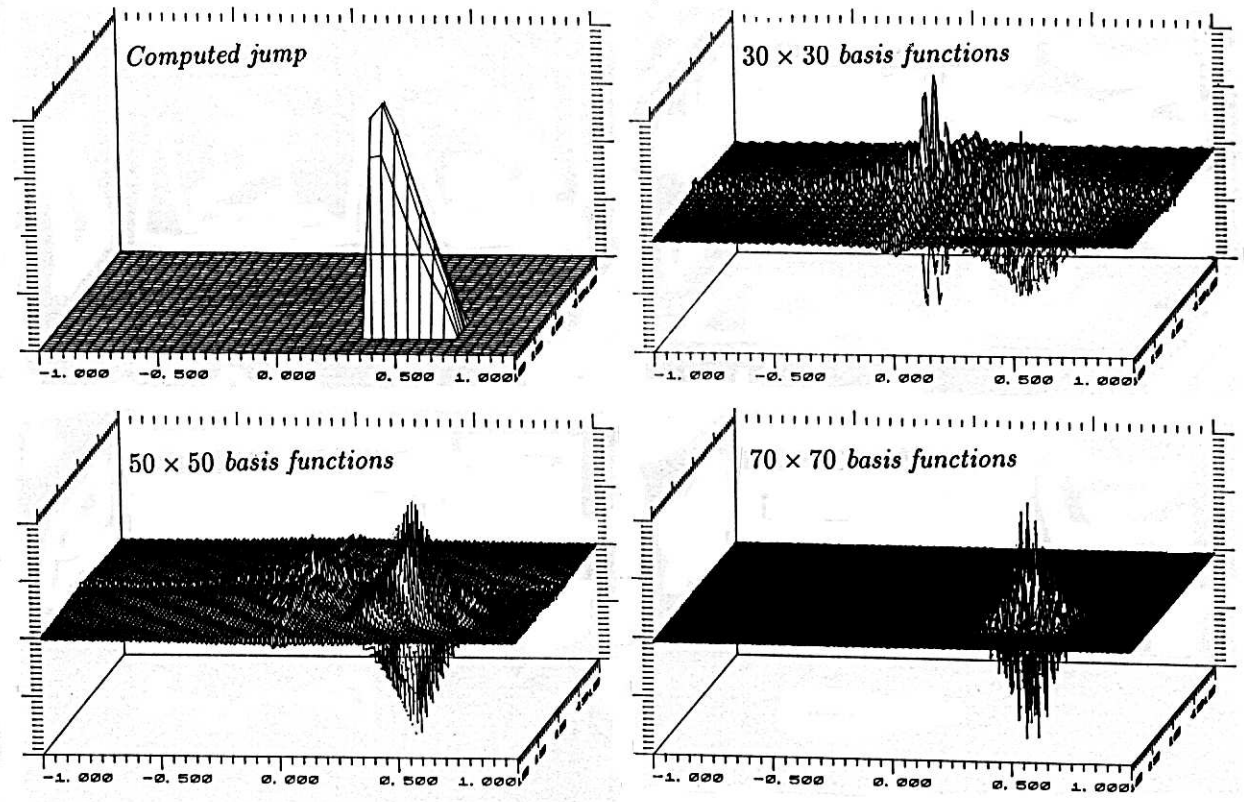

Figure 12: Reconstruction of the jump in $\mathbf{3 D}$

Using a $3 \%$ tolerance number, figure 13 shows then the reconstruction of two cracks (a special-shaped one, and a multi-crack) which establishes that the algorithm is able to reconstruct any crack, wathever its shape is. 


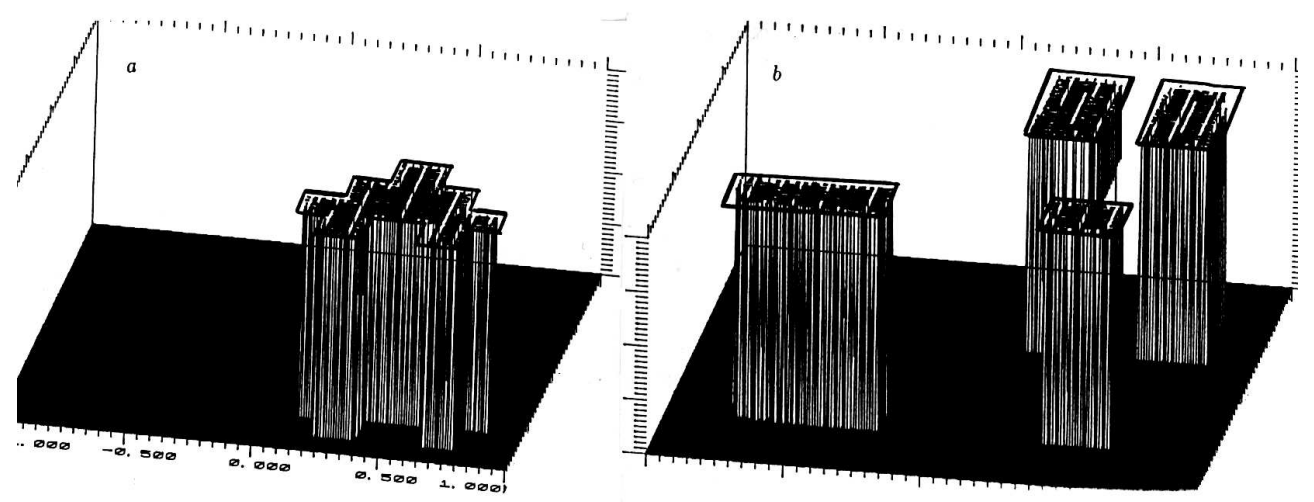

Figure 13: Reconstruction of 3D cracks a-non square crack ; b-multi crack

All the computations have been run on Sun Sparc workstations: Sparc 1 for the 2D cases, and Sparc 5 for the 3D ones. A few runs have also been performed on a Sun-Sparc 20 machine, so that it is not worthwhile to compare machine-dependent cpu times. It seems more interesting anyway to compare the time needed by the algorithm to achieve the reconstruction with the time needed by a piecewise linear finite element direct computation. Such an information is interesting because most inversion processes are iterative, and they would need, at each iteration, to solve one or several direct problems by a finite element computation. In other words, a relevant unit to compare the algorithms one to each other, in terms of computational time, would be the number of DFEC (Direct Finite Element Computations).

\begin{tabular}{|l||l||l|l|l||}
\hline & DFEC & order $30 \times 30$ & order $50 \times 50$ & order $70 \times 70$ \\
\hline \hline cpu time & $4^{\prime} \mathbf{3} 7^{\prime}$ & $9^{\prime} 22^{\prime \prime}$ & $15^{\prime} 42^{\prime \prime}$ & $19^{\prime} 31^{\prime}$ \\
\hline ratio reconstr./DFEC & & 2.03 & 3.40 & 4.23 \\
\hline
\end{tabular}

The above table gives these informations, for the reconstruction of a $3 \mathrm{D}$ single crack. It shows that the semi-explicit algorithm is a low-computer cost one, even its implementation has not been fully optimized, while the finite element code Modulef used for the computations has of course been.

\section{Conclusions}

The semi-explicit algorithm presented in this paper is based on the reciprocity gap concept, which is valid for any symmetric operator. Because of the explicit identification of the support of the crack (line in $2 \mathrm{D}$ cases, and plane in $3 \mathrm{D}$ ), the algorithm turns out to have very low computational costs, since it needs computations only on an hypersurface. Its limitations are of two types:

1. The cracks have to be planar (in 3D) or segments (in 2D), and if they are many of them, all have to be co-planar (resp. colinear). It seems difficult to go beyound such a limitation, which is constitutive of the algorithm. Recently, Santosa and Vogelius have proposed a promising lowprofile way to use the method, in order to provide a first guess for some other algorithm, when the geometric assumptions are not fulfilled. The numerical analysis of this idea has not been, up to our knowledge, done, and the compatibility conditions on the data (the prescribed and the measured ones) have also to be checked.

2. The data have to be complete, that is prescribed - or measured - on the whole external boundary. This might be a serious limitation in some practical situations, although most papers published on the topic seem to carry the same assumption.

$\operatorname{RR} n^{\circ} 3063$ 
Near-future prospects of the method lay first in its generalization to various operators, and various boundary conditions on the crack. Several results have already been obtained in this area, and some others are in progress. On another hand, it seems worthwhile to deepen the error analysis, in order to throw some additional light on the reported superconvergence phenomenon. Last, the lack of measurements on the boundary (incomplete data) could be not such a hopeless situation, at least for $2 \mathrm{D}$ cases, if a reliable way is found to couple some existing process of completing the lacking boundary data to the recovery algorithm.

Acknowledgements : The authors are indebted to Claude Zuily for the fruitful discussions they had with him on Fourier series.

\section{References}

[1] R.A. Adams : Sobolev spaces, Academic Press, 1975

[2] G. Alessandrini : Stable determination of conductivity by boundary measurements, Appl. Anal., 27 (1988), 153-172

[3] G. Alessandrini and A. Diaz Valenzuela : Unique determination of multiple cracks by two measurements, Quaderni Matematici, Università di Trieste, 1994

[4] G. Alessandrini, E. Beretta and S. Vessela : Determining linear cracks by boundary measurements; Lipschitz stability, Istituto per le applicazione del calcolo Moro Picone, CNR, 1993

[5] Sh. A. Alimov, R.R. Ashurov and A. K. Pulatov : Multiple Fourier series and Fourier integrals, in Encyclopaedia of Mathematical Sciences, 42 (Commutative harmonic Analysis IV), Springer Verlag, 1989

[6] S. Andrieux and A. Ben Abda : Identification de fissures planes par une donnée au bord unique; un procédé direct de localisation et d'identification, Comptes Rendus à l'Académie des Sciences, série 1, 315, 1992

[7] S. Andrieux and A. Ben Abda : Identification of planar cracks by complete overdetermined data : inversion formulae, Inverse Problems, 12 (1996), 553-563

[8] S. Andrieux, A. Ben Abda and M. Jaoua : Identification de frontières inaccessibles par une unique mesure de surface, Annales Maghrébines de l'ingénieur, 7, nr 1, 1993

[9] S. Andrieux, A. Ben Abda and M. Jaoua : On the emerging plane crack problem, INRIA research report nr 3012, 1996

[10] T. Bannour : Identification de fissures rectilignes ou planes par un procédé semi-explicite, DEA de Mathématiques Appliquées, ENIT, Tunis, 1995

[11] A. Ben Abda: Sur quelques problèmes inverses géométriques, Thesis, Ecole Nationale d'Ingénieurs de Tunis, 1993

[12] K. Bryan and M. Vogelius : A uniqueness result concerning the identification of a collection of cracks from finitely many electrostatic boundary measurements, SIAM J. Math. Anal., 23 (1992), 950-958

[13] K. Bryan and M. Vogelius : A computational algorithm to determine crack locations from electrostatic boundary measurements, Int. J. Eng. Sci., 32 (1994)

[14] A.R. Elcrat, V. Isakov and A. Necoloiu : On finding a surface crack from boundary measurements, Inverse Problems, 11 (1995), 343-351

[15] M. Eller : Crack detection, Inverse Problems, 12 (1996),395-408

[16] A. Friedman and M. Vogelius : Determining cracks by boundary measurements, Indiana Univ. Math. J., 38 (1989), 527-556 
[17] P. Grisvard : Elliptic problems in non smooth domains, Pitman, 1985

[18] R. Kress : Linear integral equations, Springer Verlag series in Applied Mathematical Sciences, Vol. 82, 1989

[19] F. Santosa and M. Vogelius : A computational algorithm to determine cracks from electrical boundary measurements, Int. J. Eng. Sci., 29 (1991), 917-937 
Unit'e de recherche INRIA Lorraine, Technopôle de Nancy-Brabois, Campus scientifique, 615 rue du Jardin Botanique, BP 101, 54600 VILLERS LÈS NANCY

Unit'e de recherche INRIA Rennes, Irisa, Campus universitaire de Beaulieu, 35042 RENNES Cedex

Unit'e de recherche INRIA Rhône-Alpes, 655, avenue de l'Europe, 38330 MONTBONNOT ST MARTIN

Unit'e de recherche INRIA Rocquencourt, Domaine de Voluceau, Rocquencourt, BP 105, 78153 LE CHESNAY Cedex

Unit'e de recherche INRIA Sophia-Antipolis, 2004 route des Lucioles, BP 93, 06902 SOPHIA-ANTIPOLIS Cedex

\section{Éditeur}

INRIA, Domaine de Voluceau, Rocquencourt, BP 105, 78153 LE CHESNAY Cedex (France)

ISSN 0249-6399 